\title{
7
}

\section{Motivated Humans for Reliable Software Products}

\author{
S. A. Frangos
}

Advanced Training Center Bull

26 Filellinon str., 105 58, Athens, Greece

tel.+301331-5010, fax+301331-5019,batc@prometheus.hol.gr

\begin{abstract}
This paper focuses on the software engineer, as opposed to some software engineering discipline. The author's worldwide experience in software development has resulted in concluding that the vast majority of problems encountered while developing software are more people oriented rather than technology based. Therefore, for there to be any improvements in the reliability of software, it may be wise to revisit some of the people issues, otherwise, even the best methods, tools and techniques will not make an impact on the software development process so as to result in higher levels of software quality.

Many are the problems which the software engineer is faced with while trying to piece together the complex information systems that the current global market dictates. Lack of office space and engineer concentration, unpaid overtime, non-productive meeting cultures, performance appraisals and absence of team work all contribute to the demotivation of the software engineer. Trying to introduce a new tool or a new technique to a demotivated staff is simply a waste of time. Thus, it is of paramount importance to realize that in the labor intensive software development world, the focus must first be on the human factor. Basic human nature has not changed over the years, therefore, the author sought out solutions from the past regarding the management of people, so as to be applied today in the development of software. This paper concludes with a "euphoria quadrant" so as to provide a simple means for software producing units to gauge their management style and their overall working environment.
\end{abstract}

\section{Keywords}

Hassle, humans, leadership, motivation, people, software producing units 


\section{INTRODUCTION}

"Men comprise the state and not the city walls and the ships" Thoucydides

Software Producing Units (SPUs) are faced with increased competition within the booming IT market. Successful companies achieved their competitive advantage from being able to address both the fast changing business world of today as well as the rapid pace of computer technology. Analysts try to pinpoint the exact formula for being successful in what has become a global IT market by looking into technologies, tools and techniques. Yet, the real secret behind the success or failure of an SPU lies primarily with people that make up the company or the organization. It may seem as a paradox, but technology is not the real issue in dealing with the development of high-tech information systems, but synergy amongst the people.

In dealing with the people, SPUs often treat their staff as though they are components. That is, they are treated as though they are modular in nature (DeMarco et al, 1987). The dogma "everyone is replaceable" has been adopted by most SPUs leaving a small fraction of the companies to show genuine interest for their staff. This fact alone has been catastrophic for the SPUs as software development is labor intensive. Very little of the work can be automated which translates into the SPUs being dependent on their staff for the development of quality products. The realization of this simple fact is the first basic step to improving the competitiveness of the organization.

This paper looks into the various people related issues plaguing the software development industry today. Solutions, which address the problems, will be elaborated so as to provide a winning formula for SPUs wishing to play a dominant role in a global IT market. Flashbacks to the past will be extensively employed as many of the people problems were successfully addressed in the past. It appears that the technology era has assisted in creating rigid lines between individuals, leaving the software development community to pay the high price for such a lack of human synergy. Finally, a "euphoria quadrant" is provided so that SPUs can obtain a quick assessment of their management style and working conditions.

\section{PROBLEMS}

"The sight of men whispering together in small knots or speaking in subdued tones points to disaffection amongst the rank or file" Sun Tzu

It is common for anyone from the software world to know exactly what crisis management is all about. The basic characteristic is that the SPU simply responds to identified problems and does not possess the ability to take preventive actions in order to avoid the corresponding problem areas. Companies may find themselves spending as much as $50 \%$ of their time in crisis management (Lowell, 1992). Therefore, the objective is to "de-hassle" the SPU from problems that do not allow the people to maximize synergy. This includes management as well, as they must be on the same side with the staff (Crosby, 1986). There are many contributors to what has been coined a "hassle" environment (Crosby, 1986). An analysis of the major contributors to the hassle environment will allow for a better comprehension of the issue. 


\subsection{Engineer Concentration}

Extensive studies on the subject of office space have clearly indicated that the way towards higher software development productivity is to make the working environment simply workable (DeMarco et al, 1987). It is common knowledge amongst software developers that very little work can be accomplished during normal hours. This is not surprising as software development requires high levels of concentration. During a state of concentration, significant work can be achieved, thus, the very issues of software quality and productivity are addressed by simply allowing engineers to concentrate on their work. The basic characteristic, while in a state of concentration, is that time flies. The objective here is to find the inhibitors of engineer concentration so as to eliminate or subdue them.

Incoming phone calls constitute the primary inhibitor of engineer concentration. If with every phone call a colleague receives the adjacent engineer requires an additional fifteen minutes as re-immersion to state of concentration time, it is no wonder why so many SPUs have all their engineers in a state of no concentration during normal operating hours (DeMarco et al, 1987). Most companies cluster groups of engineers in an open office environment, where there is essentially no protection against noise. Phone calls, discussions, meetings, floaters and peripherals comprise a hassle environment impeding each software developer simply trying to concentrate so as to get things done.

\subsection{Overtime}

It appears that all software managers have attended the same training course when it comes to productivity. The concept is to get more work out of the engineers per hour of pay and not per hour of work. Long hours, working weekends and holidays is all to common in our profession. An in-depth analysis of the situation indicates that overworking software professionals actually decreases productivity (DeMarco et al, 1987). Anyone who has written software code knows that one can remain productive (i.e. in a state of concentration) for only a certain amount of hours daily. Adopting the "unpaid overtime" dogma, whereby staff members are judged based on the amount of hours they spend, rather than on the quality of the produced software product, is simply catastrophic. Yet most software managers seem to relate commitment to a company with hours of unpaid overtime. It is striking that both engineer leisure time and software productivity are sacrificed for the sake of supporting the unpaid overtime dogma.

The cause of extensive overtime is also known. Software sizing is a challenging task as software requirements are not fixed early enough in the project life-cycle. Moreover, historical data regarding productivity for the selected development environment are rarely available. Therefore, unrealistic delivery dates are placed, which cannot be achieved even under the best of circumstances, leading managers to the unpaid overtime "solution".

\subsection{Meetings}

Meeting culture is considered as one of the major culprits for having demotivated staff (Crosby, 1986). Apparently, either enough meetings are not organized or they are illconducted when they do occur. Not having staff meetings invariably places management on 
the one side of the fence, while the rest of the staff are placed on the other. Management visibility regarding development as well as people issues is lost leaving intuition and information from "flatterers" as the only "raw material" upon which management decisions are made. There certainly cannot be a more demoralizing bad practice than the loss of direct contact to all levels of management for each staff member.

Ill-conducted meetings also demotivate staff. The common characteristic of an illconducted meeting is conformance to the Pareto rule. That is, management dominates $80 \%$ of the conversation leaving a mere $20 \%$ for the staff to get in a couple of words edgeways. The "faithful listener" role certainly does not have a place in software development whereby the competitiveness of the SPU is dependent on the proper exploitation of staff ideas and of the ingenuity of each and every one of the engineers.

\subsection{Appraisals}

The fact that there are so many intangible qualities attached to the development of software makes the task of conducting performance appraisals a challenge to even the best of the breed. Having observed that software developers value the quality of their work as their most important asset, performance appraisals assume the special role of judging the self-esteem of each assessed staff member. To support this observation, one simply has to take notice of the turnover rates. Upon conclusion of each appraisal period, company personnel turnover rates miraculously peak (DeMarco et al, 1987). This indicates that engineers seek recognition based on objective criteria and not based on management intuition or personal preferences (DeMarco et al, 1987).

The related issue of providing several benefits depending on company rank is also a source of demotivation. For example, policies which allow for project managers to travel in business class leaving the engineers in economy class can only be a source of problems. It is another expression of having management and staff on different sides of the wall (Crosby, 1986).

\subsection{Team Work}

SPUs have one basic characteristic in common. They never reach steady state. The business of developing software requires traversing many peaks and valleys. This inevitably means forming and breaking up project teams, as software development is project centric. Forming teams is the basic challenge for each SPU, as the team must "jell" rapidly in order to maximize the benefits from working collectively. The issue of instilling an "all in one team" cult across the project team is of paramount importance, something which has been recognized by all Total Quality Management (TQM) gurus (Logothetis, 1992). Project managers are faced with the challenge of bringing out the leading qualities of each team member and subsequently to exploit them effectively for the overall benefit of the project team.

There are a good number of reasons why teams cannot jell. The following reasons are the most common (DeMarco et al, 1987) :

- Lack of trust (defensive management)

- Paper shuffling

- Physical separation 
- Fragmentation of time

- Quality reduced product

- Phony deadlines.

\section{SOLUTIONS}

"Responsibilities for calamities are always charged to the leaders" Thoucydides

\subsection{Conformance to Requirements}

"Quality has to be defined as conformance to requirements. This definition places the organization in the position of operating to something other than opinion or experience" Philip Crosby

"Stronger is the state which has bad laws, yet applies them, rather than a state which has good laws, but doesn't apply them." Thoucydides

"Shipping, like all other trades, is difficult and cannot accommodate amateurs. Whoever is involved with this must be dedicated and must not do anything else." Thoucydides

Defining quality as conformance to requirements places the framework for operating a "hassle-free" organization. Taking the TQM principle stating that every employee has a supplier and a customer, it is easy to understand why conformance to requirements is of paramount importance regarding synergy amongst people. The essence of this definition of quality is that requirements, regarding all business processes within the SPU, must be clearly defined so that the corresponding roles have an adequate job description. That is, not only stated and implied user requirements must be extracted, analyzed and subsequently conformed to, but, all intermediate deliverables passed between software development roles must be generated based on defined procedures and standards which everyone understands and conforms to.

Assigning and enforcing roles in SPUs seems to be a difficult task. Everybody is doing everything so the IT industry has a lot of generalists and few specialists. What is sought is for meaningful roles to evolve within each SPU and to attach responsibilities to the corresponding roles. Thereafter, teams can jell around defined roles and responsibilities, so that internal as well as external customers will consistently receive products that address their stated and implied needs. Having roles and responsibilities defined, understanding what the supplier (either external or internal) will deliver and providing deliverables (either in-process or enditem) which the customer fully comprehends, leads to a workplace that is fun to be a part of.

\subsection{Institutionalizing a Quality Culture}

"Excellence is a habit. It is not an act." Aristotle

"There is nothing more difficult to take in hand, more perilous to conduct, or more uncertain in its success than to lead in the introduction of a new order of things." Nicolo Machiavelli

"If in training soldiers commands are habitually enforced, the army will be well-disciplined, if not, its discipline will be bad." Sun Tzu 
Perhaps the greatest challenge for any quality manager is to instill a quality culture. Teaching software developer "old dogs a few new tricks" requires achieving "buy-in" regarding the expected results and the means to achieve better business results. Therefore, a considerable amount of training regarding software quality, TQM, software process improvement, systematic testing, software measurements and other quality related topics, must be carried out across the entire organization, including senior management, in order to teach the new cult.

It is simply human nature to be governed by habit. There is a certain number of things that we have learned to accept, without even thinking about them. Coders seldom place comments while keying in the commands, analysts produce "cartoons" instead of diagrams that are of real use to the designers, testers refuse to make use of the techniques and tools that will allow them to fully explore all code statements and project managers make little use of meaningful software measurements so as to base their decisions on concrete data. The challenge here is to find the right people to "manage by example" so as to make new best practices a habit. This probably requires having a test specialist work with a project so as to jointly develop an effective test plan. It requires having a measurements specialist design a customized companywide metrics database and to show all levels of management how to make best use of the collected measurements. It requires for the quality department to seek out the right people to define and document the SPU's Quality Management System (QMS) so that an operational framework can be set-up for the entire staff. There is clearly a long list of what actions have to be taken. The success in instutionalization of a change is dependent on :

- Finding the right persons to envisage the required best practices;

- Implementing the changes (best practices) using a "management by example approach";

- Incorporating best practices in the SPU's QMS;

- Institutionalizing the best practices based on management's commitment not to allow exceptions or nonconformances to the documented "synergy" requirements (i.e. the QMS).

A crucial element of the above mentioned institutionalization approach is that of proper management. Actually, the use of the terms manager and leader interchangeably warrants the precise definition of the contemporary software leader by using basic concepts from the past. Unfortunately, not all managers are leaders.

\subsection{Leadership}

"The leader who knows what must be done, but cannot convince is like he does not know anything. The leader who has both characteristics, yet does not love his country, cannot give good advice." Thoucydides

"The admiral called each ship commander one by one, he reminded them of their achievements and of the fact that they had the most prestigious state as their country." Thoucydides

"If a general shows confidence in his men, but always insists on his orders being obeyed, the gain will be mutual." Sun Tzu

The software profession has matured a lot over the years and has now reached a level of maturity on the par with professions like medicine. So just as in medicine one seeks an eye doctor, if there is an eye related problem, the same is true with software. For purchasing a banking system, one must seek a supplier specializing in banking. Taking this thought a bit 
further, the supplier's knowledge in specific technologies and application areas is basically "stored" in the minds of the corresponding staff. This actually is the organization's most important asset.

A good software leader is one who understands both current technologies and has application area depth. Both are important, otherwise, the project manager cannot lead the software team through the treacherous project waves. Such vision is an extremely important acquired management quality, which means that the successful software leader is one who stays on top of technology issues and who develops in-depth knowledge in a specific application area.

It is only expected that the higher up in the organizational chart, the more management is distariced from technology issues. Therefore, in the fast moving software world it is absolutely necessary that the software leader is also a good listener so as to constantly receive input from all subordinates and to use it accordingly. The leader must trust the subordinates so they don't feel like plastic engineers simply following orders, but ones which actively participate in the decision making process (DeMarco et al, 1987). Moreover, it is expected that the software manager "wanders around" to find out first hand what the basic issues the software developers are faced with (Peters, 1985).

Senior management must seize every opportunity to instill a sense of eliteness. That is, messages such as that one should feel proud to work in the SPU and that he/she would be a fool to want to leave should be passed along in a subtle manner, but only if they are justified. A good software manager will be able to sound out problem areas just by talking to the staff. Thus, the key issue is bi-directional communication. Institutionalizing a quality culture, conformance to requirements as well as leadership capabilities in listening and telling have communication as a focal point. This is to prove that sociology, and not technology, is the culprit of most SPU problem areas (DeMarco et al, 1987).

\section{THE EUPHORIA QUADRANT}

The euphoria quadrant allows for the immediate assessment of how an SPU stands regarding the motivation of the staff. Ten (10) questions, from each of the two basic people management improvement areas, lead to the rapid assessment of the current SPU situation. The questions receive a yes/no response, with the yes receiving a one (1) point score.

\section{Work Environment}

- Is there approximately 10 square meters or more of working space per engineer ?

- Are there ( 2 meter high) partitions or walls for every two or three engineers ?

- Is electronic mail installed and in full use for all staff members ?

- Is there a phone policy (i.e. to limit phone call interruptions) ?

- Do the facilities cover basic project needs (e.g. terminals, printers, copiers, etc.) ?

- Is there a company-wide institutionalized training program?

- Do $80 \%$ of the project engineers have in-depth knowledge of the application development tools?

- Is there conformance to the company's Quality Management System?

- Does each engineer know what is expected of him/her over the next year?

- Are salaries above industry averages? 


\section{Management}

- Does senior management "wander around" ?

- Do $80 \%$ of the project managers know their application area in-depth?

- Are software delivery dates based on a costing methodology and on historical data?

- Are informal appraisals conducted ?

- Is there a significant amount of unpaid overtime ?

- Are regular meetings held (i.e. weekly project meetings, monthly department, ect.)?

- Do managers and/or specialists roll up their sleeves in order to instill a new practice?

- Do managers attend technology related courses and do they read IT periodicals ?

- Do $80 \%$ of the managers trust their subordinates (i.e. no information management)?

- Do project teams have "permanent" members (i.e. no drifters)?

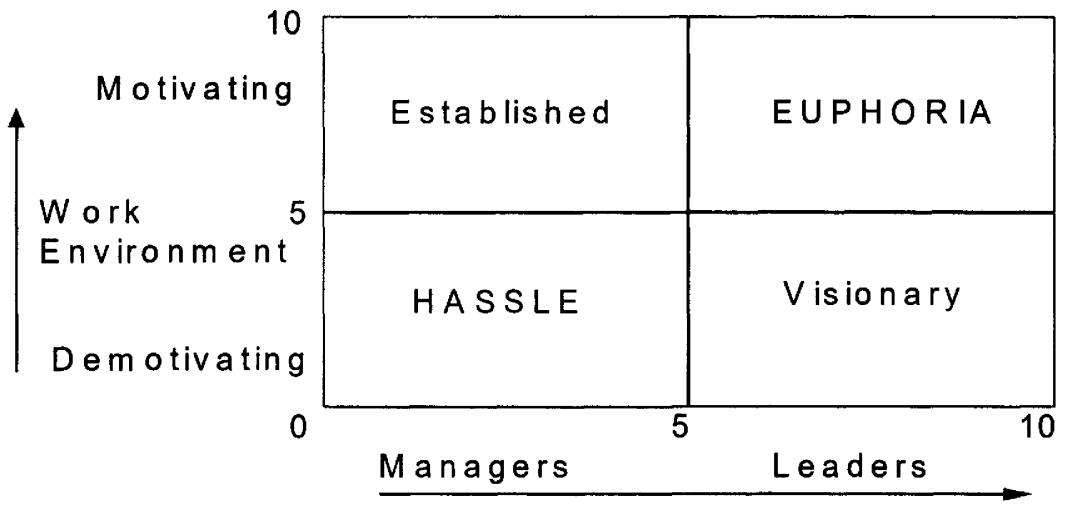

Management

Figure 1 Euphoria Quadrant.

\section{CONCLUSION}

Behind each successful software project there is certainly at least one software hero. If the software producing unit is immature, the hero is usually some charismatic coder. The more mature the organization, the less the dependence on one single hero. Thus, in a more mature SPU the project is comprised of several software heroes and the organization is one where all staff members are proud to be a part of. The essential ingredient in making the transition from a hassle environment to a euphoria environment is placing emphasis on the human factor by enhancing the working environment and by providing sound leadership.

Having leaders as opposed to simply managers, will result in at least a visionary SPU. One that is able to use the power of team work and modern technology so as to be able to stay on top of things regarding product innovation and reliability as well as continuous process improvement so as to achieve higher levels of quality. If a visionary company provides an adequate working environment, the result is a euphoria environment, one where there is 
genuine concern for the software engineer. It is possible to have successful SPUs, having poor management styles, but good working environments operating in established markets. That is, at some point such SPUs were visionaries, so as to capture a market and subsequently, collected on that "innovation". It is certain that no SPU can stay as an "established" firm for long as competition in this global IT market is fierce and innovation based on vision is a key element to success.

The Euphoria quadrant has been used to gauge several industrial with some meaningful, yet only directional results. Future work involves obtaining more industrial measurements so as to establish that software reliability is not only a function of code coverage, but, of human motivation as well.

\section{REFERENCES}

Clavell, J. 1983. The art of war (translation from Sun Tzu), Dell.

Crosby, P.B. 1984. Quality without tears : The art of hassle-free management.

DeMarco, T., Lister, T. 1987. Peopleware : Productive projects and teams, Dorset House.

Frangos, S.A. 1995. Implementing a quality management system using an incremental approach, SQM 95 Proceedings.

Logothetis, N. 1992. Managing for total quality : From Deming to Taguchi and SPC, Prentice Hall.

Lowell, A. 1992. Improving software quality : An insider's guide to TQM, Wiley.

Machiavelli, N. 1993. The Prince, Wordsworth Editions Ltd.

Peters, T. 1985. A Passion for Excellence, Random House Inc.

Vlachos, A. Thoucydides history (translation from Thoucydides), Iridanos.

\section{BIOGRAPHY}

Stelios Frangos is the general manager of the Advanced Training Center Bull, a leading Greek industrial training firm. He received a BS degree in Chemical Engineering from Rutgers (1983), USA, and a MS degree in Computer Science from NJIT (1986), USA. He is the principle designer of ISO 9001 certified QMSs for leading IT firms in Greece. He was employed in various software engineering as well as management roles worldwide and has acquired an expertise in quality engineering, methodologies, CASE tools, metrics and project management. He has delivered many seminars on software quality as well as on software project management. He is the author of several conference papers and financial journal articles on software quality. His latest interests include human resource development and modern learning practices. 